\title{
Ni extranjeras, ni secretarias: discursos de las científicas chilenas sobre el trabajo académico
}

\author{
Not Foreigners or Secretaries: Discourses of Chilean \\ Female Scientists on Academic Work
}

\section{Carla Fardella Cisternas ${ }^{1}$, Alejandra Corvalán-Navia ${ }^{1}$, Javiera Garcia-Meneses ${ }^{2}$, y Francesca Chiappini Koscina ${ }^{3}$}

${ }^{1}$ Universidad Andrés Bello, Chile.

${ }^{2}$ Pontificia Universidad Católica de Valparaíso, Chile.

${ }^{3}$ Universidad Adolfo Ibáñez, Chile.

\section{Resumen}

\begin{abstract}
Las mujeres dedicadas al trabajo científico representan menos de un tercio de quienes producen conocimiento en Chile; esto se suma a condiciones laborales desiguales, así como a una red simbólica compleja para el desarrollo científico femenino. Los crecientes estudios sobre ciencia y género tienden a centrarse en describir y analizar las condiciones y entornos académicos; sin embargo, la experiencia de las mujeres, así como sus aspectos cualitativos, han sido escasamente explorados. Este artículo reporta los hallazgos de una investigación que busca relevar la perspectiva de las científicas, indagando en los significados que construyen sobre la academia y su experiencia laboral en ella. A partir del análisis discursivo pragmático de 20 entrevistas a investigadoras de diversas disciplinas, se reportan dos repertorios interpretativos: "No quiero ser tu secretaria" y "yo soy la única mujer", y plantea los relatos de ser una minoría laboral en un orden simbólico androcéntrico. Ambos repertorios muestran como las científicas conviven creativamente con una academia que tiende a organizarse mediante prácticas que aseguran que el conocimiento producido y reproducido sea androcéntrico. Se espera que los resultados posibiliten un diálogo acerca de una academia pluralista y una comunidad científica comprometida con la justicia de género.
\end{abstract}

Palabras clave: mujer científica, ciencia y sociedad, transformación de la universidad, análisis de discurso.

\section{Correspondencia a:}

Carla Fardella Cisternas

Quillota 980, Facultad de Educación y Ciencias Sociales, Universidad Andrés Bello, Viña del Mar, Valparaíso, Chile.

carla.fardella@unab.cl

Financiamiento asociado: Proyecto Fondecyt Regular №1180129. El trabajo científico en Chile: "Instrumentos de acción pública, prácticas de producción científica e identidades laborales"; Programa de Investigación Asociativa (PIA) SOC180039; y Centro Núcleo Milenio Autoridad y Asimetrías de Poder NCS17_007.

(C) 2021 PEL, http://www.pensamientoeducativo.org - http://www.pel.cl

ISSN:0719-0409ＤDI:203.262, Santiago, Chile doi: 10.7764/PEL.58.1.2021.11 


\title{
Abstract
}

\begin{abstract}
Women who are engaged in scientific work represent less than a third of those who produce knowledge in Chile. This is in addition to unequal working conditions, as well as a symbolic network that makes it difficult for the development of female scientists. The increasing number of studies on science and gender tend to focus on describing and analyzing academic conditions and environments; however, the experiences of women, as well as their qualitative aspects, have been little studied. This paper reports the findings of a study which was intended to highlight the perspective of these female scientists, investigating the meanings that they construct regarding academia and their work experience within it. Based on the pragmatic discursive analysis of 20 interviews with female researchers from various disciplines, two interpretative repertoires are reported. The first we call "I don't want to be your secretary", which addresses the sexual division perceived by the interviewees in academic work, while the second, entitled "I'm the only woman" highlights accounts of being a minority at work in an androcentric symbolic order. The results of this research are expected to foster dialogue about pluralist academia and a scientific community committed to gender justice.
\end{abstract}

Keywords: scientific women, science and society, university transformation, discourse analysis.

La participación de mujeres en la educación superior ha tenido significativos avances en la última década, sobre todo en lo que refiere a temas de acceso (UNESCO, 2017). En muchos países, como Chile, este aumento se ve reflejado en la similitud porcentual de la matrícula de ingreso a pregrado entre hombres y mujeres (Ministerio de Educación, 2018). No obstante, los datos señalan que a medida que se avanza en los diferentes grados académicos, la presencia de las mujeres va disminuyendo (CONICYT, 2018).

En consonancia con lo anterior, cuando se observan las cifras de mujeres en la academia, solo $28 \%$ de ellas se dedica a la investigación, y luego solo 16\% lidera equipos científicos de alto rendimiento (CONICYT, 2017). Como resultado, las académicas y, específicamente, las científicas, se encuentran subrepresentadas a nivel de rectorías, secretarías generales, direcciones de posgrado e investigaciones, así como también, en menor medida, en cargos vinculados a vicerrectorías y decanaturas académicas (Berríos, 2007; Del Pino, Vallejos, AmésticaRivas, y Cornejo-Saavedra, 2018; Gaete-Quezada, 2015; Kiss, Barrios, y Álvarez, 2007).

Estos datos evidencian un escenario desigual no solo en términos de cifras, sino que muestran también un escenario simbólicamente adverso para las científicas. En efecto, el aumento de mujeres en educación terciaria y su creciente inserción en la ciencia no implica que la trama simbólica acerca de las diferencias de género organizadas en el espacio científico se esté modificando. De acuerdo con Pacheco, Cayeros y Navarro (2011), la rigidez de la trama simbólica podría estar dada por la supuesta cultura de objetividad y neutralidad de la academia y la ciencia. Sin embargo, esta supuesta objetividad no sería más que la generalización de valores androcéntricos que han sido construidos y conceptualizados como valores neutrales y universales.

En relación con lo anterior, si bien una serie de estudios que aborda la temática de mujeres y ciencia describe las condiciones laborales, y pone en evidencia las brechas en torno a presencia, reconocimiento y remuneración de las científicas en la academia, su experiencia laboral cotidiana ha sido escasamente investigada. En consecuencia, resulta fundamental comprender los significados y discursos que construyen las mujeres que habitan la ciencia respondiendo a la pregunta acerca de cómolas científicas describen la academia y su experiencia laboral en ella. 
A partir del análisis discursivo pragmático de 20 entrevistas activas (Denzin, 2001) a científicas de diversas disciplinas (Ingeniería Comercial, Sociología, Física, Educación Parvularia, Educación Diferencial, Antropología, Psicología, Química Ambiental, Filosofía, Ingeniería Civil, Trabajo Social, Leyes e Historia), se obtuvieron dos repertorios interpretativos centrales para comprender cómo las científicas construyen los significados respecto de su experiencia laboral en la academia.

\section{Revisión del panorama de las mujeres científicas en Chile}

\section{Cifras y brechas de la participación femenina en la academia}

Según la UNESCO (2017), las mujeres conforman el 28,8\% del total de investigadores a nivel mundial y un 45,4\% a nivel latinoamericano. En Chile, el porcentaje de mujeres en investigación es de 31,1\%, posicionando a nuestro país en el puesto 17 de 20 países consultados en Latinoamérica y el Caribe. En concordancia, según el informe estadístico emitido por la Unidad de Estudios de la Comisión Nacional de Investigación Científica y Tecnológica (2018) - de ahora en adelante, CONICYT -, si bien las mujeres acceden en mayor o igual proporción que los hombres a educación de pre y posgrado (53,1\% y 50,2\%, respectivamente), estas cifras disminuyen a medida que se avanza en la carrera académica.

En línea con lo anterior, a nivel de pregrado, el porcentaje de mujeres tituladas supera al de los hombres (56,6\%); en posgrado, en cambio, el porcentaje disminuye, con un $48 \%$ en programas de magíster y $43,5 \%$ en doctorados (CONICYT, 2018). Luego, por diversos motivos, quienes se dedican a investigación solo corresponden a 28\% (UNESCO, 2017). Este porcentaje persiste y se reitera en la adjudicación de becas posdoctorales, Iniciación y también Regular, en donde el porcentaje de mujeres que se adjudican proyectos Fondecyt, FONDAF y PIA no supera el 35\% del total en cada uno de los proyectos. En el caso de los proyectos EXPLORA, FONIS y Regional, la situación cambia, y la adjudicación de mujeres a estos proyectos es mayor que la de los hombres $-51 \%, 59,1 \%$ y $63,6 \%$, respectivamente (CONICYT, 2018)-.

Respecto de la participación femenina en la academia, considerando las 60 universidades identificadas en el reporte de personal académico 2018 del Servicio de Información de Educación Superior (SIES), se observa que $43,4 \%$ del total de académicos son mujeres; sin embargo, este porcentaje decrece a medida que aumenta el rango etario en el cual se encuentra dicho personal (CONICYT, 2018). Asimismo, si bien las mujeres representan cerca del $45 \%$ de las plantas docentes, y $35 \%$ de los profesores asistentes son mujeres, solo un 30\% está contratado por jornada completa, y solo 15\% de los profesores titulares son mujeres (Universidad de Chile, 2014).

Por otro lado, se observa una disminución en el porcentaje de participación femenina en la academia a medida que la cantidad de dinero y poder a distribuir crece. Si se miran los escalafones académicos, entendidos como "los espacios o casilleros que ocupan los docentes en virtud de los antecedentes curriculares, la antigüedad docente y los méritos profesionales" (Kiss et al., 2007, p. 97), encontramos una disminución de la representación de las mujeres en los escalafones más altos. En este sentido, solamente $20 \%$ de las instituciones de educación superior y consejos científicos son encabezados por una mujer (considerando directores, vicerrectores y rectores), y solo 8,2\% de las rectoras son mujeres (Observatorio de Género en Educación Superior, 2019).

Así, en el contexto laboral académico nos encontramos con mujeres dedicadas preferentemente a la docencia y con una minoría enfocada a fortalecer los indicadores que posibilitan el ascenso en la jerarquía (Kiss et al., 2007). Si bien en el área de investigación 31,5\% de los investigadores son mujeres, el porcentaje de investigadoras disminuye al avanzar en roles de mayor cargo: mientras que 45\% del Personal Técnico y de Apoyo son mujeres, solo hay 16\% de directoras de Centros de Investigación tipo Fondap, PIA y Milenio (CONICYT, 2017). 
Todas las brechas mencionadas se acentúan cuando las vinculamos a los salarios recibidos y su diferenciación según género. Según el Instituto Nacional de Estadística (INE), por hora de trabajo científico e intelectual los ingresos de las mujeres son inferiores a los de los hombres en un 34\% (INE, 2017). Como señala el informe, parte de esto se explica por las diferencias de remuneraciones entre facultades: aquellas con menor presencia femenina tienen las remuneraciones más altas (Universidad de Chile, 2014).

\section{Más allá de las cifras: discursos y representaciones de lo femenino en la academia}

Más allá de las cifras expuestas, es importante profundizar en las diferentes interpretaciones y explicaciones que ha construido la literatura especializada en torno a las brechas e inequidades antes mencionadas. Tal como exponen Kiss y sus colaboradores (2007), la educación superior y la ciencia se han configurado históricamente como un espacio adverso y ajeno para las mujeres. Esto se asocia a la predominancia fundacional de varones, que ha fomentado una cultura androcéntrica con múltiples efectos en estructuras formales, representaciones simbólicas y prácticas no formales, que se despliegan a diario en las instituciones universitarias.

El androcentrismo, como señala Saini (2017), permea la cultura académica; sin embargo, suele quedar escondido tras los discursos de neutralidad, objetividad y pluralismo bajo los cuales promete haberse fundado. Es decir, aun cuando la academia se ha construido bajo la promesa de ser un lugar para la reflexión y el progreso, lo cierto es que las representaciones de lo masculino y lo femenino responden con naturalidad y sin mayor complejización a los discursos de la cultura dominante androcéntrica extraacadémica.

Como señalamos, los discursos acerca de lo femenino y lo masculino en la academia tienden a reproducir los estereotipos de género anclados en la cultura hegemónica en al menos dos sentidos: por un lado, una serie de estudios documentan la clásica división sexual (trabajo de cuidado y trabajo productivo) y, por otro, las atribuciones de inferioridad/superioridad cognitiva atribuidas a mujeres y hombres, respectivamente. Cabe mencionar que hemos trabajado con la noción de discurso (Potter, 1998) y no de estereotipos de género, ya que permite entender que las nociones de lo masculino y lo femenino no son solo esquemas mentales en torno a un objeto externo, sino más bien una construcción colectiva (no mental-interna) que, además, está dotada y sostenida por relaciones y prácticas de poder específicas de su contexto (Tipler \& Ruscher, 2019), permitiéndonos abordar el problema de la mujer en la ciencia de manera más compleja y anclada socialmente.

El discurso acerca de la división sexual del trabajo hace referencia a la discusión planteada en los años 70 respecto de cómo las tareas y roles sociales se han dividido en función del sexo, asignando a los hombres las tareas productivas, remuneradas y públicas y, en el otro extremo, las tareas de reproductivas, de cuidado, no remuneradas y privadas a las mujeres (Morton, 1970; Benston, 1997). En el plano académico, diferentes estudios muestran cómo esta división se reitera: las tareas de gestión, cuidado del personal o atención de estudiantes tienden a estar centralizadas en las académicas (Muñiz, 2016; Müller, 2019; Saracostti, 2006; Tronto, 1993), mientras que las tareas de consecución de recursos y fondos de investigación y representación de proyectos suelen tener un rostro masculino (Ríos, Mandiola, y Varas, 2017). Frente a esto, Muñiz (2016) señala cómo los roles asociados al cuidado dentro de las facultades - principalmente la formación y la administración de la vida cotidiana- han quedado subordinados a la investigación, innovación y cargos de representación, discurso que se asocia en gran medida al llamado suelo pegajoso, que se explicará más adelante.

Por otro lado, encontramos los discursos relativos a la inferioridad/superioridad cognitiva organizados bajo la premisa de que las mujeres son más emocionales y los hombres, más racionales (Morton, 1970; Benston, 1997; Saini, 2018). En esta línea, algunos autores muestran cómo las representaciones de lo masculino y femenino tienden a ser polares: lo masculino se asocia a nociones de competencia, asertividad, autoridad y orientación al logro, mientras que lo femenino se vincula a calidez, sociabilidad, emotividad y orientación a las relaciones (Glinsner, Sauer, Gaitsch, Penz, 
\& Hofbauer, 2017; Hochschild, 2003). En el plano del trabajo académico, esto se traduce en la creencia de que las mujeres tienen dificultades para ejercer cargos de autoridad, puesto que son inseguras o no son capaces de imponer orden o tomar decisiones racionalmente (Barberá, Candela, y Ramos, 2008; Jost \& Kay, 2005; Quiles et al., 2008).

Los discursos en torno a lo femenino/masculino y cómo estos operan en el plano académico se encuentran indefectiblemente asociados a los fenómenos ampliamente descritos en los estudios que abordan mujeres, ciencia y trabajo (Martínez-Labrín y Bivort-Urrutia, 2014). Uno de los fenómenos que caracterizan esta división se expresa metafóricamente como techo de cristal: "Esta estructura se usa para explicar el freno del ascenso de las mujeres en un cierto nivel debido a factores invisibles -informales- en los sistemas de evaluación y promoción" (Martínez-Labrín y Bivort-Urrutia, 2014, p. 17). El techo de cristal hace referencia al freno que se impone a las mujeres para ascender profesionalmente, producto de características que se adjudican tácitamente al género femenino; no obstante, el techo de cristal, si bien es una práctica invisible, es suficientemente frecuente como para constituir uno de los fenómenos centrales en la experiencia de las mujeres en el campo laboral (Rebolledo, 2001).

Las barreras que obstaculizan el avance de las mujeres en sus caminos profesionales no se dan únicamente de modo vertical, sino también de forma horizontal. Para dar cuenta de este fenómeno, la literatura ha hablado de los muros de cristal, los cuales expresan "la dificultad que presentan las mujeres para cambiar de un área a otra de trabajo o moverse horizontalmente dentro de su organización, por ejemplo, cambiar desde docencia a investigación” (Martínez-Labrín y Bivort-Urrutia, 2014, p. 17). Por ejemplo, las mujeres tienden a tener mayor presencia en las áreas feminizadas y subvaloradas del conocimiento, como educación, enfermería, psicología o trabajo social, áreas que por lo demás están asociadas al discurso del cuidado y el trabajo reproductivo. Además del techo y muros de cristal, está el piso pegajoso, noción que intenta dar cuenta de cómo las mujeres en la academia tienden a tomar roles de gestión y tareas de mantenimiento subvaloradas por la organización universitaria, lo que tiene como efecto aplanar sus carreras y entorpecer su ascenso hacia puestos de poder (Del Pino et al., 2018; Martínez, 2012; Ríos et al., 2017).

Los discursos y fenómenos subyacentes a la desigualdad en la participación de las académicas se han visto tensionados y visibilizados, en parte, por el mayor acceso de las mujeres al contexto laboral universitario, pues su creciente presencia ha fisurado los discursos, normas e institucionalidad laboral vigentes en los espacios académicos (Yáñez, 1999). Sin embargo, todo lo anterior muestra que el orden disciplinario y androcéntrico continúa, posicionando a las científicas como intrusas en la academia (Berlien, Varela, y Robayo 2016; MartínezLabrín y Bivort-Urrutia 2014; Ríos et al., 2017).

Es necesario comprender que el escenario académico androcéntrico, su orden simbólico, sus valorizaciones del trabajo y representaciones acerca de qué es la ciencia (Fardella, 2020; Fardella, Sisto, y Jiménez, 2015) se han plasmado en prácticas cotidianas visibles e invisibles, que facilitan una cultura académica centrada en lo masculino, favoreciéndoles por sobre otras identidades académicas, y relegando a las mujeres a una posición marginal y cargada de ambigüedad y silencios (Berríos, 2008; Del Pino et al., 2018; Harding, 1997; Longo, 2009). En este sentido, este trabajo propone relevar la perspectiva de las científicas, indagando en los discursos y significados que ellas construyen sobre la academia y su experiencia laboral en ella.

\section{Método}

Para abordar adecuadamente los objetivos planteados para este estudio, se optó por un enfoque metodológico cualitativo (Denzin \& Lincoln, 2003; Flick, 2004). Esta perspectiva resulta particularmente apropiada para identificar los discursos que las mujeres científicas de Chile han construido con base en su experiencia laboral, en un contexto de rediseño e implementación de políticas públicas de orientación managerial para la productividad científica. 
Entre los años 2016 y 2018 se realizaron 20 entrevistas activas (Denzin, 2001) a mujeres académicas de la zona central del país, representantes de diferentes áreas del conocimiento, con altas tasas de publicaciones científicas y adjudicación de fondos concursables. Esto con el propósito de comprender discursos construidos respecto de su entorno laboral, su oficio y su desempeño dentro de este. Estos datos están vinculados a un estudio más amplio sobre la transformación de la ciencia en Chile (Fardella-Cisternas, Sisto, y Jiménez, 2017; Fardella, Corvalán, y Zavala, 2019).

Para obtener los datos, se realizaron entrevistas activas, definidas como una interacción en la cual los participantes (entrevistador/a y entrevistado/a) son asumidos como sujetos que interactúan abiertamente (Denzin, 2001), aunque guiadas temáticamente por una pauta flexible con formato de respuesta abierta, que se organizó en torno a tres temas específicos: trayectorias científicas, prácticas y experiencias en la producción científica, e identidad laboral. La flexibilidad y espontaneidad de la entrevista activa lleva necesariamente a espacios discursivos nuevos, que hace que entrevistadas y entrevistadoras se vean en la necesidad de relatar y reorganizar nuevos relatos acerca de sus quehaceres (Denzin \& Lincoln, 2003).

Cada entrevista fue grabada y transcrita, utilizando el sistema Jefferson (Potter, 1998), que permite conservar características activas del habla. A continuación, se explican los símbolos utilizados:

- Texto en mayúsculas indica grito o expresiones más sonoras que el resto.

- Texto subrayado indica palabras o partes de palabras que son acentuadas por el hablante.

- Flecha hacia abajo precede a una marcada baja de la entonación.

- Flecha hacia arriba precede a una marcada subida de la entonación.

- Dos puntos indican la prolongación del sonido inmediatamente anterior. La cantidad de veces que se repiten los dos puntos indica la extensión de la prolongación.

- Número entre paréntesis indica la duración, en segundos, de una pausa en el habla.

- Un punto entre paréntesis indica una pausa breve, normalmente menos que 0,2 segundos.

El corpus total de las entrevistas se codificó en función del contenido, creando unidades de significado filtradas y reorganizadas en forma abierta mediante técnicas cualitativas de análisis de contenido. Estas se basan en la extracción de ideas clave que permiten articular la información en categorías, las cuales cumplen el papel de hipótesis, que son contrastadas en momentos posteriores al análisis o integradas en categorías más complejas (Strauss \& Corbin, 1990). En un primer paso, las entrevistas fueron fragmentadas y simplificadas en códigos que permitieron una gestión simple del volumen de datos. Esta fase culminó con un libro de 47 códigos, agrupados en unidades temáticas sometidas al análisis de discurso pragmático y al análisis de repertorios interpretativos (Potter, 1998). Este trabajo reporta aquellos códigos y categorías que refieren específicamente a los discursos en torno a la experiencia laboral.

\section{Resultados}

Con el objetivo de responder a la pregunta sobre cómo las mujeres científicas describen la academia y su experiencia laboral en ella, los resultados que se presentan a continuación se dividen en dos grandes categorías. La primera categoría llamada "No quiero ser tu secretaria" refiere a la división sexual del trabajo académico y la subvaloración que tienen las tareas que las mujeres realizan dentro de este espacio laboral, relatando cómo han enfrentado esta situación. La segunda categoría, denominada "Yo soy la única mujer", refiere a la racionalidad masculina que norma la academia y que ha llevado a que las mujeres habiten este espacio desde un lugar de extranjería. A su vez, analizamos cuáles son los efectos de estos discursos en su posicionamiento en el actual 
contexto académico, un escenario predominantemente masculino en la educación superior: el orden simbólico y las representaciones que derivan de él, junto con las practicas cotidianas, visibles e invisibles, facilitan que el varón construya una subjetividad masculina anclada culturalmente en su capacidad de adquirir prestigio y una posición jerárquica superior, mientras que las mujeres habitan una posición marginal y cargada de ambigüedad y silencios.

\section{"No quiero ser tu secretaria": la división sexual del trabajo académico}

Las académicas describen la universidad como un escenario donde el trabajo académico se organiza según categorías binarias de género. Respecto de esto, los discursos analizados develan que, en la mayoría de los casos, las mujeres asumen y se les designan tareas y roles ligados a la gestión y a la atención de estudiantes de sus respectivas escuelas o facultades; a su vez, a los varones se les confieren las labores de investigación o representación de la facultad. Las entrevistas muestran cómo esta organización del trabajo académico se desarrolla espontáneamente, sin una planificación explícita en los departamentos.

Entro a la: sala, me siento y me miran todos y me dice, 'Cata te vamos a tener que pedir un favor es que no tenemos traducción simultánea. (0.2)' Tuve que traducir toda la conversación (...) PUROS HOMBRES, y yo:: yo tuve que TRADUCIR, ¡saben lo que es eso? (0.2) O sea::, hablaba en inglés y yo lo decía en español, hablaba alguien en español y yo le traducía al inglés (Entrevista 10).

Este fragmento ilustra cómo la organización del trabajo académico reproduce el modelo de división sexual del trabajo de manera natural y mecánica. A partir de las entrevistas, es evidente que las mujeres en la universidad asumen labores asociadas a las funciones domésticas y de servicios. En este escenario, las labores relegadas a las académicas parecieran vincularse dócilmente al trabajo emocional (Hochschild, 2003), asunto que, en el discurso hegemónico de los roles de género, aparece como el contrapunto de la racionalidad y neutralidad que caracterizaría el trabajo científico. Coherentemente, los académicos varones aparecen vinculados a estas tareas. Respecto de esto, una de las entrevistadas señala:

En las escuelas hay muchas de estas secretarias acadé:micas que resuelven toda:s, todos estos problemas domé:sticos, ¿sí? Y: y: los hombres como están más como en esos cargos ya:: (0.1) me encargo del grupo de investigación, ¿ya? O: me encargo de otra cosa no: yo no puedo estar en ese comité y yo no entiendo: ¿̨m:? (Entrevista 2).

Cabe destacar que las tareas distribuidas y asignadas a varones y mujeres en la academia no solamente son diferentes y se asocian dócilmente al modelo de división sexual del trabajo, sino que además se trata de tareas a las que se le asignan diferente valor. Asociado a esto, las mujeres se enfrentan a que las tareas que les son asignadas prioritariamente reciban menos reconocimiento o incluso sean invisibles para los sistemas estandarizados de evaluación del desempeño (Fardella-Cisternas et al., 2017; Fardella et al., 2019). Muchas entrevistadas refirieron a escenas de este tipo y mostraron su desagrado frente a esto:

Estábamos acá sentados, haciendo un informe y él se para y se pone atrás y me empieza a dicta:r ((risas)). Entonces le dije ya no sabis qué, estay reproduciendo demasiado la escena del gerente con la secretaria, me in:cómoda, siéntate aquí al lado mejor ((risas)). (Entrevista 20).

Tal como señalan Martínez y Bivort (2014), este tipo de situaciones aluden a lo que se ha definido como segregación vertical, entendida como la construcción de los límites simbólicos para establecer una frontera entre lo femenino y lo masculino en los espacios productivos. Esto provoca la asociación binaria y jerarquizada, donde el polo de arriba es masculino, productivo, racional y reconocido, mientras que el polo de abajo es femenino, doméstico, afectivo e invisible. 
(...) Yo no quiero trabajar como su secretaria, ¿no? Es como: si no fueran capaces de utilizar la compu, bueno, mala suerte, yo no puedo hacerlo (0.1), ahí se necesita igualdad en condicio:nes para poder trabajar (Entrevista 12).

Tal como lo advierte el fragmento, la disimulada pero persistente división sexual del trabajo académico es reconocida por las académicas como condiciones desiguales. En efecto, esta distribución hace que ellas ocupen su tiempo en labores de menor valor, disminuyendo su tiempo de escritura, en desmedro de la productividad científica, provocando en muchos casos la exclusión silenciosa de las tareas que traen más prestigio, como la investigación (Berríos, 2008). Tal como señalan Ríos et al. (2017), las académicas constatan que las labores administrativas provocarían un "modo de organizar su inserción a la universidad que boicotea sus carreras académicas" (p. 120).

para mí, yo creo que es un buen equilibrio dar clases y compartir con alumnos mi pasión y hacer proyectos con alumnos, y también tener algo de tiempo, porque al final no me queda tanto, quizás un día a la semana, para mis proyectos de investigación (Entrevista 3).

Las escenas relatadas son, de alguna manera, una exposición de la subjetividad femenina a la subvaloración de su trabajo, lo cual cuestiona la imagen de sí como trabajadora. En los relatos, se ven tensionados y se dificultan los modos en que las entrevistadas construyen el sentido de su trabajo. Frente a la desvalorización de sus tareas en la academia y la posición de desventaja, las hablantes buscan reconstruir positivamente el sentido de su trabajo. En esta dimensión, para algunas mujeres, el vínculo con los estudiantes - derivado sobre todo de las actividades de docencia o de formación de investigadores- es relatado como un recurso central para incrementar el valor de su labor. La docencia, en tanto actividad de indiscutible y consensuada trascendencia social, permite a las hablantes recuperar la apreciación de su rol. Acerca de esto, las académicas señalan:

al final no teni la misma productividad que tus colegas ho::mbres (.) ahora, lo que es interesante es que apareci como un modelo siginificati:vo eh: $p a{ }^{\prime}$ cierto grupo de estudiantes (Entrevista 10).

En resumen, las académicas describen su contexto laboral como un espacio en el que existe una evidente división del trabajo asociada a los roles de género, puesto que a las mujeres se les delegan, generalmente, las labores de servicio y administración por sobre las labores de investigación. Esta división del trabajo académico no solo implica una segmentación de tareas, sino que también una jerarquización de estas. Muchas entrevistadas declaran la desvalorización de las labores de servicio y administración que les son atribuidas, al mismo tiempo que una dificultad u obstaculización para dedicar tiempo a las tareas que implican mayor prestigio simbólico. Frente a esta tensión, los relatos ponen a la docencia y el reconocimiento de sus estudiantes como un elemento central, para restituir la importancia de su rol. De acuerdo con Rodríguez (2014), la lucha por darle sentido al trabajo en un contexto laboral que devalúa la imagen de la trabajadora se da mediante discursos éticos y afectivos (Pullen, Rhodes, \& Thanem, 2017), ya que el valor del trabajo no siempre puede ser alcanzado por criterios tradicionales, como la riqueza, el estatus y la autoridad.

\section{"Yo soy la única mujer": el orden simbólico androcéntrico en el trabajo masculino}

"La única mujer" es un recurso retórico ilustrativo que evidencia cómo las académicas construyen su entorno laboral a la vez que se constituyen a sí mismas como una minoría. Esto, a su vez, tiene el efecto de mostrar el contrapunto con la presencia mayoritaria de varones dentro de las universidades y centros de investigación. Es así como para las entrevistadas, ser la única mujer es un criterio relevante para describir y describirse en su escenario laboral.

yo soy la única mujer. y la secretaria (.) Claro y: no hay otras profes. No, acá en filosofía, yo soy la única mujer (Entrevista 11). 
Si bien en muchas disciplinas los académicos constituyen la mayoría, también es cierto que hay muchas otras disciplinas donde ellas no son minoría (especialmente las denominadas feminizadas, como enfermería, pedagogía, psicología). Aun así, las mujeres continúan percibiendo el espacio laboral como masculino, tal como se ilustra en el siguiente fragmento:

la educación es un campo de mujeres; sin embargo, los que estamos citando siempre son hombres (Entrevista 6).

La construcción de las hablantes como minoría se articula cómodamente, con el concepto extranjería, desarrollado por Martínez (2015), que permite conceptualizar la participación de las mujeres en la academia como una experiencia de foraneidad o fuera de lugar.

Efectivamente, algo se da cuando somos puras mujeres, que sentí una libertad, una confianza, decí lo que te da la gana y: no hay filtro, ¿me entendí? Y en el fondo seguramente, ellos (.) siempre se mueven así (...) los hombres seguramente siempre han vivido así. Lo que nosotros es una asamblea de cien mujeres, que no hay ningún hombre (...) ellos siempre han vivido (0.1) naturalmente, en esa sensación de: sensación (...) esa sensación de: comodidad (...) ellos no necesitan crear esa: esa: lo artificial de estar (.) (...) lo normal $p a^{\prime}$ ellos es eso (Entrevista 6).

Los relatos dan cuenta de cómo la sensación de extranjería se construye discursivamente como contrapunto a la sensación de comodidad de los varones en la academia. Es frecuente en los relatos describir experiencias cotidianas de sometimiento, desconfianza, incomodidad y rechazo, como experiencias opuestas a lo que los hombres experimentan en sus contextos laborales. De esta forma, los relatos en torno a la sensación de exclusión son abundantes en las entrevistas:

ellos cambiaron el horario de la reunión a un horario fuera del horario laboral, y yo quedé fuera porque tenía que ir a cuidar a mi hijo (Entrevista 8).

no había reuniones regulares, o sea no había espacios explícitos, comunes y formales para desarrollar el trabajo (...) el trabajo se hacía a través de las relaciones que ellos tenían establecidas, su relación de amista::d previa, eh: SUS canales de comunicación personales y yo nunca estaba ahí (Entrevista 10).

Bueno, las decisiones políticas se toman en otro lado ¿no? (...) esos dos tipos, colegas académicos, decidieron no sé: tomando un café cuando yo no estaba presente (Entrevista 2).

La experiencia de extranjería relatada no solo tiene relación con ser una minoría numérica, sino que devela discursivamente el reconocimiento de una norma extrańa, derivada de un orden androcéntrico. Y es justamente la extranjería lo que permite la distancia para poder reconocer aquella gramática que les es ajena a las mujeres. Esto ha sido denominado por una de las hablantes como racionalidad masculina, relacionada con lo que ha sido descrito por autoras como comunidades epistémicas patriarcales (Harding, 1997; Haraway, 1995).

cuando me decían que yo pensaba como un hombre:era siempre un piropo (.) eso (.) Porque: me estaban diciendo que era muy se::ria: que: que: que podía argumentar bie:n o sea: (.) y YO me sentía bien, yo me sentía halagada. Yo: empecé a estudiar temas que filosóficamente eran temas tremendamente oscu:ros y difi:ciles y por lo tanto eran los que yo quería porque: era lo que: ME HACÍA VALIDARME también, ¿̨no? (Entrevista 17).

La racionalidad masculina es una figura que ilustra la distancia que perciben las mujeres con la gramática académica, a la vez que evidencian la posible y potencial existencia de otros lenguajes académicos. Como menciona la entrevistada: 
Como cápsulas de realidad, así se construye el conocimiento, ese es el conocimiento que prima, si querí decir algo así teni que lograr ser escuchada por esos tipos, porque, por esas personas que están construyendo ese tipo de conocimiento (Entrevista 2).

En este sentido, la organización del trabajo académico y la gramática subyacente a la forma de construir el conocimiento se rige por normas androcéntricas, que suelen ser un referente para ordenar los otros discursos presentes en la academia. Respecto de esto, algunas académicas explicitan cómo han debido aprender estas lenguas para poder ser escuchadas por sus colegas. En efecto, esta impostura es narrada como una estrategia que les permitiría a las hablantes encontrar o conseguir un disfraz para habitar un espacio de (cierta) autoridad en sus contextos laborales. En línea con esto, la experiencia muestra cómo las mujeres perciben que la academia en general, pero también la producción de conocimiento en particular, tiende a organizarse bajo prácticas que aseguran que el conocimiento producido y reproducido sea masculino.

\section{Discusiones finales}

Los resultados presentados dan cuenta de cómo las académicas describen su contexto y experiencia laboral, evidenciando que esta última está íntima y profundamente marcada por los discursos acerca de lo masculino y femenino en el campo académico. De acuerdo con los relatos, las académicas identifican prácticas sutiles de discriminación en una serie de ámbitos laborales, como la producción en investigación, los escalafones académicos y la división de las tareas académicas, en donde ellas son relegadas a labores administrativas por sobre las de mayor prestigio, como la investigación.

A pesar de lo anterior, las académicas le han otorgado un significado particular a las labores que han sido desvalorizadas en los contextos académicos en los que trabajan, otorgándoles un carácter axiológico femenino. De esta manera, estas mujeres muestran cómo es posible resignificar su experiencia de académicas y científicas, reivindicando sus condiciones laborales, llegando a ascender en su trayectoria, logrando así la consolidación laboral y personal.

Las proyecciones de este estudio guardan relación con la necesidad de continuar explorando aquellos aspectos idiosincráticos de la experiencia femenina en la academia. Desde ahí, los afectos aparecen como relevantes tanto en la construcción identitaria de las académicas como en las prácticas académicas cotidianas. Los afectos podrían estar asociados a prácticas androcéntricas, donde por un lado la extranjería se asocia a la inseguridad y la auto atribución de insuficiencia en el contexto académico. De esta manera, como plantean Pullen y sus colaboradoras (2017), es a través de los encuentros cotidianos y las relaciones con otros que los afectos se mueven entre los cuerpos y se asientan como órdenes sociales, que o bien dotan de capacidades o limitan a los cuerpos femeninos. Así, mediante la repetición de ciertos afectos desplegados en eventos mundanos, se generan registros afectivos y, en cada interacción, se les propone a las sujetas cómo se siente ser académica. Por otro lado, los afectos también pueden movilizar relaciones íntimas, de colaboración y confianza entre las académicas, que agencian la experiencia y transforman las maneras previstas de ser y estar en la academia. De este modo, el poner el acento en estos fenómenos puede potenciar la creación de comunidades epistémicas diferentes, que problematicen las formas actuales de organización competitiva e híper individualizada de la universidad.

Hasta aquí, se ha evidenciado que la sola inclusión de mujeres no asegura la construcción de una universidad pluralista e inclusiva. Por ello, también es necesario explorar fenómenos vinculados a barreras de clase y etnia, que visibilicen las tensiones y exclusiones de mujeres provenientes de contextos con menores ingresos socioeconómicos o de contextos rurales o de pueblos originarios. Por esto, es necesario expandir y explorar cómo otras subjetividades disidentes a la figura de académico hombre/blanco construyen versiones de mundo que navegan la academia actual. 
El artículo original fue recibido el 2 de febrero de 2020

El artículo revisado fue recibido el 8 de marzo de 2020

El artículo fue aceptado el 10 de junio de 2020

\section{Referencias}

Barberá, E., Candela, C., y Ramos, A. (2008). Elección de carrera, desarrollo profesional y estereotipos de género. Revista de Psicología Social, 23(2), 275-285. https://doi.org/10.1174/021347408784135805

Benston, M. (1997). Political Economy of Women's Liberation. En R. Hennessy \& C. Ingraham (Eds), Materialist Feminism: A Reader in Class, Difference, and Women's Lives (pp. 17-23). Londres: Routledge.

Berlien, K., Varela, P., y Robayo, C. (2016). Realidad Nacional en Formación y Promoción de Mujeres Cientificas en Ciencia, Tecnología, Ingeniería y Matemáticas. Santiago, Chile: CONICYT. Recuperado de https:/www.conicyt.cl/wp-content/uploads/2016/12/Estudio-Realidad-Nacional-en-STEM.pdf

Berríos, P. (2007). Análisis sobre las profesoras universitarias y desafíos para la profesión académica en Chile. Calidad en la Educación, (26), 39-53. https://doi.org/10.31619/caledu.n26.232

Berríos, P. (2008). Carrera académica: análisis empírico de su estructura y organización en Chile. Calidad en la Educación, (29), 36-62. https://doi.org/10.31619/caledu.n29.187

Comisión Nacional de Investigación Científica y Tecnológica (CONICYT). (2017). Diagnóstico Igualdad de Género en Ciencia, Tecnología e Innovación en Chile. Levantando evidencias, construyendo avances y proponiendo recomendaciones desde la colaboración pública y privada. Santiago, Chile: Departamento de Estudios y Gestión Estratégica. Recuperado de https://www.conicyt.cl/wp-content/uploads/2015/03/Diagnostico-Equidad-deGenero-en-CTI-MESA-CONICYT_2017.pdf

Comisión Nacional de Investigación Científica y Tecnológica (CONICYT). (2018). Mujeres en Ciencia. Santiago, Chile: CONICYT.

Del Pino, S., Vallejos, R., Améstica-Rivas, L., y Cornejo-Saavedra, E. (2018). Presencia de las mujeres en la alta gestión universitaria. Las universidades públicas en Chile. Páginas de Educación, 11(2), 176-198. https://doi.org/10.22235/pe.v11i2.1633

Denzin, N. (2001). The reflexive interview and a performative social science. Qualitative Research, 1(1), 23-46. https://doi.org/10.1177/146879410100100102

Denzin, N. \& Lincoln, Y. (2003). Strategies for qualitative inquiry. Thousands Oaks: Sage.

Fardella, C. (2020). Abrir la jaula de oro. La universidad managerial y sus sujetos. Revista Izquierdas, 49, 2299-2320. Recuperado de http://www.izquierdas.cl/images/pdf/2020/n49/art110_2299_2320.pdf

Fardella, C., Sisto, V., y Jiménez, F. (2015). Nosotros los académicos. Narrativas identitarias y autodefinición en la universidad actual. Universitas Psychologica, 14(SPE5), 1625-1636. https://doi.org/10.11144/Javeriana.upsy14-5.nani

Fardella-Cisternas, C., Sisto, V., y Jiménez, F. (2017). La transformación de la universidad y los dispositivos de cuantificación. Estudos de Psicologia (Campinas), 34(3), 435-448. https://doi.org/10.1590/1982-02752017000300011

Fardella, C., Corvalán, A., y Zavala, R. (2019). El académico cuantificado. La gestión performativa a través de los instrumentos de medición en la ciencia. Psicología, Conocimiento y Sociedad, 9(2), 62-78. https://doi.org/10.26864/pcs.v9.n2.15

Flick, U. (2004). Introducción a la investigación cualitativa (1a edición). Madrid, Espańa: Morata.

Gaete-Quezada, R. (2015). El techo de cristal en las universidades estatales chilenas. Un análisis exploratorio. Revista iberoamericana de educación superior, 6(17), 3-20. https://doi.org/10.1016/j.rides.2015.06.001

Glinsner, B., Sauer, B., Gaitsch, M., Penz, O., \& Hofbauer, J. (2017). Doing gender in public services: affective labor of employment agents. Gender Work Organization, 26(7), 983-999. https://doi.org/10.1111/gwao.12263

Haraway, D. J. (1995). Ciencia, cyborgs y mujeres: la reinvención de la naturaleza. Valencia, España: Ediciones Cátedra.

Harding, S. (1997). Ciencia y feminismo. Madrid, España: Morata.

Hochschild, A. (2003). The managed heart. California, CA: University of California Press. 
Instituto Nacional de Estadísticas (INE). (2017). Enfoque estadístico: Género e Ingresos 2016 (archivo PDF). Recuperado de https://www.ine.cl/docs/default-source/genero/documentos-de-an\%C3\%A1lisis/documentos/genero_ ingresos_2017.pdf?sfvrsn=94daef_8

Jost, J. T. \& Kay, A. C. (2005). Exposure to benevolent sexism and complementary gender stereotypes: consequences for specific and diffuse forms of system justification. Journal of personality and social psychology, 88(3), 498-509. Recuperado de https://psycnet.apa.org/buy/2005-01818-006

Kiss, D., Barrios, O., y Álvarez, J. (2007). Inequidad y diferencia. Mujeres y desarrollo académico. Revista Estudos Feministas, 15(1), 85-105. https://doi.org/10.1590/S0104-026X2007000100006

Longo, E. (2009). Género y trayectorias laborales. Un análisis del entramado permanente de exclusiones del trabajo. Trayectorias: revista de ciencias sociales de la Universidad Nacional de Nuevo León, 28, 118-141. Recuperado de http://trayectorias.uanl.mx/28/index.htm

Martínez-Labrín, S. y Bivort-Urrutia, B. (2014). Procesos de producción de subjetividad de género en el trabajo académico: Tiempos y espacios desde cuerpos femeninos. Psicoperspectivas, 13(1), 15-22. https://doi.org/10.5027/psicoperspectivas-Vol13-Issue1-fulltext-334

Martínez, S. (2012). Ser o no ser: tensión entre familia, subjetividad femenina y trabajo académico en Chile: Un análisis de desde la psicología feminista. Revista de estudios de género, 4(35), 133-163. https://doi.org/10.32870/lv.v4i35.726

Martínez, S. (2015). Género, subjetividad y trabajo académico. Integración Académica en Psicología, 3(8), 47-53. Recuperado de http://www.integracion-academica.org/attachments/article/91/Integraci\%C3\%B3n\%20 Acad\%C3\%A9mica\%20en\%20Psicolog\%C3\%ADa\%20V3N8\%20red\%202a.pdf\#page $=50$

Ministerio de Educación. (2018). Estadísticas de la Educación 2017. Santiago, Chile: MINEDUC.

Morton, P. (1970). El trabajo de la mujer nunca se termina. En M. Henault, P. Morton, \& I. Larguia (Eds.), Las mujeres dicen basta (pp. 41-67). Buenos Aires, Argentina: Nueva Mujer.

Müller, B. (2019). The carelesss society - dependency and care work in capitalist societies. Hypothesis and Theory, 3, 44. https://doi.org/10.3389/fsoc.2018.00044

Muñiz , L. (2016). Narrativas femeninas sobre el mundo del trabajo: reflexiones en torno al trabajo de las mujeres en sectores masculinizados del mercado laboral. Papeles de Trabajo - Centro de Estudios Interdisciplinarios en Etnolingüistica y Antropología Socio-Cultural, 31, 65-78. Recuperado de http://rephip.unr.edu.ar/handle/2133/12785

Observatorio de Género en Educación Superior. (2019). Visualización de datos universidades. Recuperado de https://equidaddegenero.mineduc.cl/observatorio-de-genero-en-educacion-superior.html

Pacheco, L., Cayeros, L., y Navarro, C. (2011). El dispositivo sexo/ ciencia. Revista venezolana de estudios de la mujer, 16(36), 31-46. Recuperado de http://saber.ucv.ve/ojs/index.php/rev_vem/article/view/2023

Potter, J. (1998). La representación social de la realidad. Discurso, retórica y construcción social. Barcelona, España: Paidós.

Pullen, A., Rhodes, C., \& Thanem, T. (2017). Affective politics in gendered organizations: affirmative notes on becoming-woman. Organization, 24(1), 105-123. https://doi.org/10.1177/1350508416668367

Quiles, M. N., Morera, M. D., Correa, A. D., Navas, M., Gómez-Berrocal, C., y Cuadrado, I. (2008). El prejuicio hacia las mujeres:¿ infrahumanización o infravaloración? Revista de Psicología Social, 23(2), 221-228. https://doi.org/10.1174/021347408784135797

Rebolledo, L. (2001). Impacto de los estudios de género en la democratización de las instituciones de Educación Superior. Revista de estudios de género: La ventana, 2(13), 75-93. https://doi.org/10.32870/lv.v2i13.552

Ríos, N., Mandiola, M., y Varas, A. (2017). Haciendo género, haciendo academia: Un análisis feminista de la organización del trabajo académico en Chile. Psicoperspectivas, 16(2), 114-124. https://doi.org/10.5027/psicoperspectivas-vol16-issue2-fulltext-1041

Rodríguez, J. (2014). Labors of Love: Nursing Homes and the Structures of Care Work. Nueva York, NY: NYU Press.

Saini, A. (2018). Inferior: cómo la ciencia infravalora a la mujer y cómo las investigaciones reescriben la historia. España: Ediciones Círculo de Tiza.

Saracostti, M. (2006). Mujeres en la alta dirección de educación superior: posibilidades, tensiones y nuevas interrogantes. Calidad en la Educación, (25), 243-259. https://doi.org/10.31619/caledu.n25.261

Strauss, A. \& Corbin, J. (1990). Basics of qualitative research. Sage publications. 
Tipler, C. N. \& Ruscher, J. B. (2019). Dehumanizing representations of women: the shaping of hostile sexist attitudes through animalistic metaphors. Journal of Gender Studies, 28(1), 109-118. https://doi.org/10.1080/09589236.2017.1411790

Tronto, J. (1993). Moral boundaries. Londres, Reino Unido: Routledge.

UNESCO. (2017). Women in Science. Fact Sheet No 43 (archivo PDF). Recuperado de http://uis.unesco.org/sites/ default/files/documents/fs43-women-in-science-2017-en.pdf

Universidad de Chile. (2014). Del Biombo a la Cátedra: Igualdad de Oportunidades de Género en la Universidad de Chile. Santiago, Chile: Universidad de Chile. Recuperado de http://web.uchile.cl/archivos/VEX/BiomboCatedra/ files/assets/basic-html/index.html\#1

Yañez, S. (1999). Consideraciones sobre flexibilidad laboral planteadas desde una mirada de género. En J. Ensignia \& S. Yáñez (Eds.), Sindicalismo, género y flexibilización en el Mercosur y Chile. Inserción laboral femenina (pp. 97-110). Santiago, Chile: Fundación Friedrich Ebert. 